\title{
Bensmaine $\mathrm{F}^{1}$, Le Mapihan $\mathrm{K}^{1}$, Defrance $\mathrm{F}^{1}$, Lion $\mathrm{G}^{3}$, Ernst $\mathrm{O}^{4}$, Pigny $\mathrm{P}^{5}$, Vantyghem MC ${ }^{1,2}$
}

1 Department of Endocrinology and Metabolism, Lille University Hospital, Lille, France. 2 INSERM, U859 Biotherapies for Diabetes, Lille, France. 3 Department of Nuclear

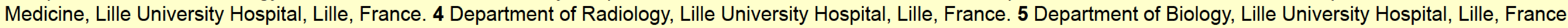

INTRODUCTION Irisin is a myokine, which displays a day-night rhythm, is correlated with lean body mass, and increases after exercise in healthy young individuals, despite an association with major adverse cardiovascular events and polycystic ovary disease (Mantzoros 2014). Increased skeletal muscle volume has been reported in women with familial partial lipodystrophies (FPLD) (Ji JCEM 2014). Our aim was to to determine whether irisin could be a marker of FPLD, which is characterized by a decreased fat mass and an increased lean mass (Clin.gov2009-AO-1169-48/PHRC 2009_09/094).

\section{METHODS AND PATIENTS}

Circulating irisin levels (EIA Phoenix) were measured in :

- 20 LMNA-related FPLD,

- 19 healthy normal-weighed $(\mathrm{H})$ and

- 13 obese non-diabetic (OND) patients

And correlated with :

- body composition (assessed with DEXA and MRI),

- metabolic parameterd (fasting blood glucose (FBG),

- insulin, A1C, lipid, transaminases, leptin) and

- inflammatory markers (leukocytes CD4, CD8, CRP)

Values were expressed as median IQR and were compared wih the non-parametric Kruskal-Wallis test (Statview). Correlations were studied by the non-parametric Spearman correlation test. The Wilcoxon non-parametric test was used to compare biological data between H, FPLD, and OND.

RESULTS Irisin median differed significantly between the 3 groups (p:0.0076), was higher in OND ( $p: 0.0099)$ and FPLD ( $p: 0.047$ ) than in $\mathrm{H}$ groups, without any difference between FPLD and OND and was similar between male and female.

Irisin was not correlated with leptin or inflammatory markers. Leptinemia was higher in OND compared to $\mathrm{H}$ and FPLD $(p<0.0001)$, without difference between these 2 groups. Irisin/leptin ratio, a biomarker of lean/fat mass, was lower in OND (21(13-32)) than in FPLD (166 (71-214)) or control $(164(128-222))$ groups $(p<0.0001$ without difference between FPLD and $\mathrm{H}$.

\begin{tabular}{|c|c|c|c|c|c|c|c|c|c|c|}
\hline & H(1) & {$\left[Q_{1}, Q_{2}\right]$} & $\begin{array}{l}\text { OND } \\
\text { (2) }\end{array}$ & {$\left[Q, Q_{2}\right]$} & FPLDI & {$\left[Q_{2}, Q_{2}\right]$} & $P_{12 t+23^{3}}$ & $\mathrm{P}_{1 / 1^{*}}$ & $\mathrm{P}_{1 / 3^{*}}$ & $\mathrm{P}_{2 / 3^{*}}$ \\
\hline Number (n) & 19 & & 13 & & 20 & & & & & \\
\hline Age (years) & 37 & [23-55] & 51 & [21-56] & 45 & [33-58.5] & 0.22 & $\cdot$ & . & $\cdot$ \\
\hline Weight (kg) & 69 & [55-76.8. & 100.4 & [94-104] & 67.3 & [55.6-80.75] & 0.0001 & 0.0001 & 1.000 & 0.0001 \\
\hline BMI $\left(\mathrm{kg} / \mathrm{m}^{2}\right)$ & 22.3 & [21-24.2.2] & 39 & [36.8-41] & 25.6 & [21.97-28.5] & $<0.0001$ & $<0.0001$ & 0.1 & 0.0001 \\
\hline Fasting blood glucose (g//) & 0.86 & {$[0.83 \cdot 0.94]$} & 0.92 & {$[0.83-0.99]$} & 1.06 & [0.89-1.51] & 0.0084 & 0.87 & 0.009 & 0.19 \\
\hline Fasting blood insulin(g//) & 4.5 & {$[3.1-5.8]$} & 7.3 & [4.5-10.55] & 9.3 & [6.4-18.4] & 0.001 & 0.07 & 0.001 & 0.84 \\
\hline Hemoglobin Alc $(\%)$ & 5 & [4.9.5.5.6] & 5.8 & {$[5.46 .2]$} & 6.15 & {$[5.6-7]$} & $<0.0001$ & 0.0033 & 0.0001 & 0.45 \\
\hline \multicolumn{11}{|l|}{ Transaminases (U//L) } \\
\hline SGOT & 22 & {$[19-25]$} & 20 & {$[19-25]$} & 32 & [23.5.-39] & 0.0059 & 1.000 & 0.02 & 0.03 \\
\hline SGPT & 17 & [14-25] & 20 & [19-27] & 29 & [225-52] & 0.004 & 0.089 & 0.009 & 0.33 \\
\hline \multicolumn{11}{|l|}{ Lipids (g)/1 } \\
\hline Total cholestereral & 1.95 & [1.8-2.12] & 1.88 & [1.75-2.13] & 1.76 & [1.59-2.12] & 0.34 & . & . & $\cdot$ \\
\hline Timglycerides & 0.88 & [0.62:-1.16] & 1.31 & [1.07-1.59] & 2.14 & {$[1.08-2.66]$} & 0.0015 & 0.05 & 0.004 & 0.23 \\
\hline Lepting (ng/m) & 4.6 & [4.1:10.7] & 49.6 & [31.9.67.6] & 6.8 & [4.15-11.95] & $<0.0001$ & $<0.0001$ & 1.000 & 0.0001 \\
\hline lising (ng/mi) & 804 & [732-876] & 934 & [872-1046] & 940 & [810-1066] & 0.0076 & 0.006 & & \\
\hline $\operatorname{CRP}(\mathrm{mg} / /)$ & 0 & 0 & 7 & {$[3 \cdot 15]$} & 3 & {$[0.0 .3 .5]$} & 0.0001 & $\langle 0.0001$ & 0.0009 & 0.11 \\
\hline
\end{tabular}

Tab 1 : Median distribution of metabolic and inflammatory parameters in healthy controls, obese non diabetic and FPLD patients.

\begin{tabular}{|c|c|c|c|c|c|c|c|c|c|c|}
\hline & $\mathrm{HC}^{(1)}$ & {$\left[Q_{1}, Q_{3}\right]$} & OND $(2)$ & {$\left[Q_{1}, Q_{3}\right]$} & FPLD & {$\left[Q_{1}, Q_{3}\right]$} & $p_{1+2+3^{*}}{ }^{*}$ & $p_{1 / 2}{ }^{*}$ & $p_{1 / 3}{ }^{*}$ & $p_{2} /{ }^{*}$ \\
\hline \multicolumn{11}{|l|}{ Fat mass (DEXA) } \\
\hline Total fat mass (\%) & 22.7 & {$[20-30.7]$} & 45.4 & [43.9.9-48.9] & 20.75 & [16.75-28.15] & $<0.0001$ & $<0.0001$ & 0.99 & $<0.0001$ \\
\hline Fat mass $/$ height $\left(\mathrm{g} / \mathrm{m}^{2}\right)$ & 0.52 & {$[0.43-0.87]$} & 1.78 & {$[1.64-2.02]$} & 0.58 & {$[0.34-0.05]$} & $<0.0001$ & $<0.0001$ & 1.000 & $<0.0001$ \\
\hline \multicolumn{11}{|l|}{ Lean mass (DEXA) } \\
\hline Lean Mass (\%) & 73.38 & {$[66-76.4]$} & 52.4 & [48.75-54.25] & 75.66 & [68.55-79.88] & $<0.0001$ & $<0.0001$ & 0.99 & $<0.0001$ \\
\hline Lean mass $/$ height ${ }^{2}\left(\mathrm{~g} / \mathrm{m}^{2}\right)$ & 1.64 & {$[1,5-1.8]$} & 2.06 & {$[1,91-2.2]$} & 1.8 & {$[1,7-2,13]$} & $<0.0001$ & $<0.0001$ & 0.08 & 0.07 \\
\hline Total fat mass/ total lean mass & 0.31 & {$[0.26-0.5]$} & 0.87 & {$[0.81-1]$} & 0.29 & {$[0.21-0.44]$} & $<0.0001$ & $<0.0001$ & 1.000 & $<0.0001$ \\
\hline \multicolumn{11}{|l|}{ Abdominal fat mass (MRI) } \\
\hline Total abdominal fat mass (cc) & 276 & {$[166-374]$} & 833 & [711.5-937.5] & 216.5 & [153.5-310.5] & $<0.0001$ & $<0.0001$ & 1.000 & $<0.0001$ \\
\hline Intra abdominal fat mass (cc) & 50 & [21-92] & 182.5 & [1222.5-336] & 124.5 & [65-164.5] & 0.0002 & 0.0006 & 0.03 & 0.13 \\
\hline $\begin{array}{l}\text { Intra abdominal fat mass/ Total } \\
\text { abdominal fat mass }\left(\mathrm{g} / \mathrm{m}^{2}\right) /(\mathrm{cc})\end{array}$ & 0.2 & {$[0.11-0.3]$} & 0.25 & {$[0.15-0.33]$} & 0.57 & {$[0,46-0.7]$} & 0.0001 & 0.74 & 0.0006 & 0.0075 \\
\hline $\begin{array}{l}\text { Lean mass } / \text { height }{ }^{2} / \text { intra } \\
\text { abdominal fat fass }\left(\mathrm{g} / \mathrm{m}^{2} \mathrm{l}\right) /(\mathrm{cc})\end{array}$ & 0.03 & {$[0.02-0.07]$} & 0.01 & {$[0.01-0.02]$} & 0.02 & {$[0.01-0.022]$} & 0.0005 & 0.0015 & 0.04 & 0.1 \\
\hline $\begin{array}{l}\text { Lean mass } / \text { heighth2/ total } \\
\text { abdominal fat mass }\left(\mathrm{g} / \mathrm{m}^{2}\right) /(\mathrm{cc})\end{array}$ & 0.006 & {$[0.004-0.010]$} & 0.002 & {$[0.002-0.003]$} & 0.009 & {$[0.006-0.012]$} & $<0.0001$ & $<0.0001$ & 0.36 & $<0.0001$ \\
\hline
\end{tabular}

Tab 2: Median distribution of anthropometrical parameters in healthy controls, obese non diabetic and FPLD patients.

\begin{tabular}{|c|c|c|}
\hline & $r$ & $\mathrm{p}$ \\
\hline Weight $(\mathrm{kg})$ & $+0,386$ & 0,005 \\
\hline $\mathrm{BM}\left(\mathrm{kg} / \mathrm{m}^{2}\right)$ & $+0,453$ & 0,0008 \\
\hline Fasting blood glucose (gil) & $+0,330$ & 0,017 \\
\hline Fasting blood insulin $(g / l)$ & $+0,421$ & 0,002 \\
\hline Hemoglobin A1C (\%) & $+0,349$ & 0,012 \\
\hline SGPT (UI/L) & $+0,333$ & 0,016 \\
\hline Total cholesterol (g/l) & $+0,397$ & 0,0039 \\
\hline Triglycerides $(g / l)$ & $+0,494$ & 0,0002 \\
\hline Lean massiheight ${ }^{2}\left(g / m^{2}\right)$ - DEXA & $+0,517$ & $<0,0001$ \\
\hline |A fat mass (cc) - MRI & $+0,349$ & 0,017 \\
\hline |A fat mass/TA fat mass - MRI & +0.39 & 0.0069 \\
\hline
\end{tabular}

Tab 3: Correlation between irisin and metabolic, inflammatory and anthropometrical markers $\mathrm{IA}=$ intra-abdominal, $\mathrm{TA}=$ total abdominal

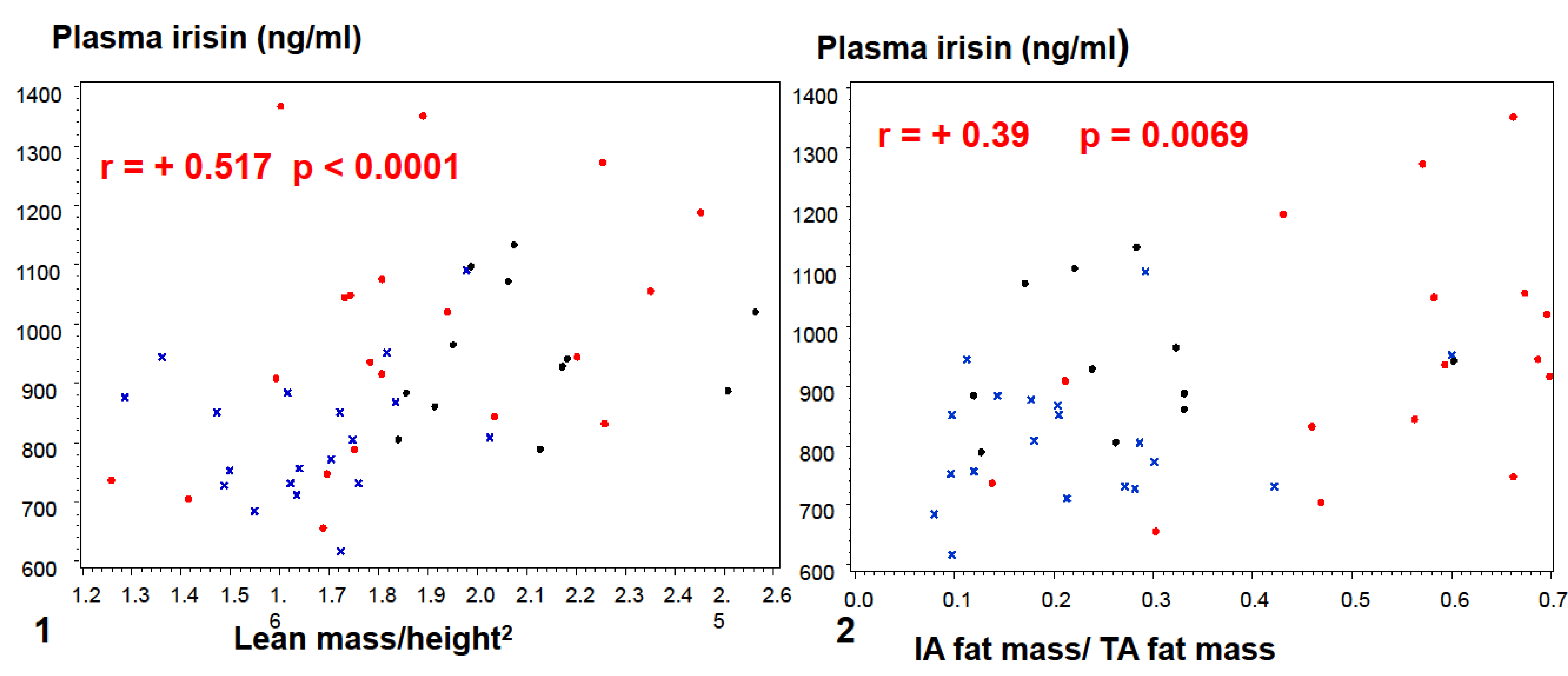

Fig 1 : Correlation of irisin and LEFT lean mass/height ${ }^{2}$ in the whole population and RIGHT IA / TA fat mass, HC, OND, FPLD

CONCLUSION Compared to control, FPLD is characterized by high irisinemia and similar leptinemia, OND by both high leptinemia and irisinemia. Irisin is increased in diseases characterized by higher lean mass whatever the amount of fat mass. 